\title{
Structural basis of heparan sulfate-specific degradation by heparinase III
}

\author{
Wei Dong ${ }^{1}$, Weiqin Lu ${ }^{2,3}$, Wallace L. McKeehan ${ }^{2}$, Yongde Luo ${ }^{2}$, Sheng Ye ${ }^{1 凶}$ \\ ${ }^{1}$ Life Sciences Institute, Zhejiang University, Hangzhou 310058, China \\ ${ }^{2}$ Center for Cancer and Stem Cell Biology, Institute of Biosciences and Technology, Texas A\&M Health Science Center, \\ Houston, Texas 77030, USA \\ ${ }^{3}$ Department of Molecular Pathology, The University of Texas MD Anderson Cancer Center, Houston, Texas 77030, USA \\ $\triangle$ Correspondence: sye@zju.edu.cn (S. Ye) or yluo@ibt.tamhsc.edu (Y. Luo) \\ Received June 5, 2012 Accepted June 17, 2012
}

\begin{abstract}
Heparinase III (HepIII) is a 73-kDa polysaccharide lyase (PL) that degrades the heparan sulfate (HS) polysaccharides at sulfate-rare regions, which are important co-factors for a vast array of functional distinct proteins including the well-characterized antithrombin and the FGF/FGFR signal transduction system. It functions in cleaving metazoan heparan sulfate (HS) and providing carbon, nitrogen and sulfate sources for host microorganisms. It has long been used to deduce the structure of HS and heparin motifs; however, the structure of its own is unknown. Here we report the crystal structure of the Heplll from Bacteroides thetaiotaomicron at a resolution of $1.6 \AA$. The overall architecture of HepllI belongs to the $(\alpha / \alpha)_{5}$ toroid subclass with an $\mathrm{N}$-terminal toroid-like domain and a $\mathrm{C}$-terminal $\beta$-sandwich domain. Analysis of this high-resolution structure allows us to identify a potential HS substrate binding site in a tunnel between the two domains. A tetrasaccharide substrate bound model suggests an elimination mechanism in the HS degradation. Asn260 and His464 neutralize the carboxylic group, whereas Tyr314 serves both as a general base in C-5 proton abstraction, and a general acid in a proton donation to reconstitute the terminal hydroxyl group, respectively. The structure of HepllI and the proposed reaction model provide a molecular basis for its potential practical utilization and the mechanism of its eliminative degradation for HS polysaccarides.
\end{abstract}

KEYWORDS heparinase III, crystal structure, heparan sulfate, fibroblast growth factor (FGF), $\beta$-elimination

\section{INTRODUCTION}

Polysacharides are important components of extracellular matrix and on the cell membrane where a variety of biological events take place (Kjellen and Lindahl, 1991; Ren et al., 2011). Heparin/heparan sulfate (HS) glycosaminoglycans (HSGAGs) are the representatives of theses biological polysaccharides (Jackson et al., 1991). They recognize specific molecules and play critical roles in various biological processes, such as signaling pathways (Bornemann et al., 2004; Han et al., 2004; Takei et al., 2004) and development (Perrimon and Bernfield, 2000; Bulow and Hobert, 2006). One major function of $\mathrm{HS}$ is to interact with both fibroblast growth factor (FGFs) and receptors (FGFRs), and to form productive FGF-HS-FGFR signaling complexes (Rapraeger et al., 1991; Yayon et al., 1991; Kan et al., 1993; Ye et al., 2001; Kamimura et al., 2006; Luo et al., 2006). The involvement of structurally specific HS motifs as cofactor in the FGF signaling system suggests that the activity and specificity of the FGF system may be modulated by HS and in turn by the enzymes that degrade or synthesize HS (Guimond and Turnbull, 1999; Kan et al., 1999; Harmer, 2006; Luo et al., 2006; Lamanna et al., 2008; Zhang et al., 2009). Beyond the FGF/FGFR system, HS has other significant values in its participation in diverse biological functions of an array of other distinct proteins (Capila and Linhardt, 2002).

HS, as a heterogeneous mixture, displays a large diversity in both physical and chemical properties and in specific biological activities; however, these HS molecules share a similar backbone structure and consist of basic disaccharide repeats of uronic acid (UA) and glucosamine $(\mathrm{GlcN})$ that are arranged in a linear fashion (Maccarana et al., 1996; Sugahara and Kitagawa, 2002). Both HS and heparin un- 
dergo various sulfation modifications on different locations after the initial synthesis to various degrees; as a result, they possess commonly a highly negatively charged property (Esko and Selleck, 2002). Heparin restricts most of the UA to iduronic acid (IdoA) and exhibits a high degree of sulfation. Heparan sulfate, on the other hand, takes the form of glucuronic acid (GICA) with a significantly low level of sulfation. The main modification patterns of heparin and HS are reported to be the 2-O-sulfation on uronic acid, 6-O-sulfation, 2-N-sulfation/acetylation and the rare 3-O-sulfation on glucosamine (Ernst et al., 1995; Sasisekharan and Venkataraman, 2000). The lower level of sulfation imparts HS to a less negatively charged state compared to heparin and significantly more structural heterogeneity, therefore, more diversity in specific motifs that interact with a vast array of distinct proteins.

Previous studies have shown that two distinct mechanisms are employed by HS-degrading enzymes including the microbial heparinase family and the mammalian heparanase, to depolymerize the GAGs (Yip and Withers, 2004). Microbial heparinases are known to achieve the degradation by the so-called $\beta$-elimination mechanism, in which an acidic group is considered in a position to neutralize the negative charge of uronic acid, and a basic group acts as a marauder to abstract the proton from the $\mathrm{C}-5$ atom (Scheme 1). Efforts from both of the acid and the base ultimately result in an unsaturated double bond formation between C-4 and C- 5 at the non-reducing end (Linhardt et al., 1986; Peter, 1987; McCarter and Withers, 1994). In contrast to any of the heparinases, the hydrolytic mechanism is mainly utilized by the mammalian heparanase to catalyze the HS depolymerization in a water-dependent way, in which the intermediate disaccharide is not to create a double bond between C-4 and C-5 of uronic acid residue, but to be preferentially accepted by a water molecule. Eventually, a saturated disaccharide is produced (Kussie et al., 1999; Toyoshima and Nakajima, 1999).

Microbial heparinases include three main types, heparinase I, II and III. Although all of them are eliminases that primarily act on the glycosidic bond between the uronic acid and glucosamine, releasing saturated or unsaturated disaccharide products, they show considerably a low degree of similarity in primary sequences and differ from each other in multiple aspects of chemical and activity properties (Lohse and Linhardt, 1992; Godavarti and Sasisekharan, 1996). Heparinase I (Hepl; EC 4.2.2.7) is a heparin lyase with a molecular weight of $42.8 \mathrm{kDa}$ and displays a preference toward highly sulfated regions of heparin as its main substrate (Desai et al., 1993a, b). The selective cleavage occurs at glycosidic linkage of GlcN2S6S(1-4)IdoAp2S within the sulfation-rich domain. 2-O sulfation of IdoA is recognized as an essential element for the scission; therefore, the poorly sulfated HS is mostly excluded by heparinase I. In a previous study, we described the expression and high-yield purification of active hepainase I from mammalian gut symbiont Bacteroides thetaiotaomicron that can be used for large scale structural analysis of HS and heparin motifs in the aids of either PAGE gel or mass spectrometer (Luo et al., 2007). Heparinase II (Hepll; EC not assigned) with a molecular weight of $84.1 \mathrm{kDa}$ has a broad selectivity to both heparin and $\mathrm{HS}$, provided that the uronic acid is modified by a 2-O-sulfate group (Nader et al., 1990; Moffat et al., 1991; Desai et al., 1993a, b). Heparinase III (HepIII; EC 4.2.2.8) with a molecular weight of $70.8 \mathrm{kDa}$, on the other hand, was reported to have a restricted substrate specificity in catalyzing HS degradation (Desai et al., 1993a, b). It has a unique ability to cleave the $\alpha(1-4)$ glycosidic bond between GlcN and GlcA, as $\mathrm{GlcA}(1-4) \mathrm{GlcNAc}$, provided that the GlcA moiety at $\mathrm{C} 2$ position is not sulfated. In fact, it has been found that the sulfation-poor region of HS provides the high affinity binding site for Heplll to make the reaction carry out efficiently and selectively. As different types of heparinases have different substrate specificity, there have been important aids in elucidating the complex structures of heparin and HS motifs. In addition, their therapeutic utility is emerging. Heplll is reported to be a potent inhibitor of neovascularization (Sasisekharan et al., 1994). Although the atomic structures and recombinant preparation of active heparinase I and II have promoted our understanding of their enzymatic modes of action and substrate specificity, a similarly representative structure and a large-scale preparation for heplll are still lacking. Here we report the recombinant expression, enzymatic characterization and the crystal structure of heparinase III in a ligand-free state and propose a potential active site of degradation reaction to elucidate its possible molecular mechanism.
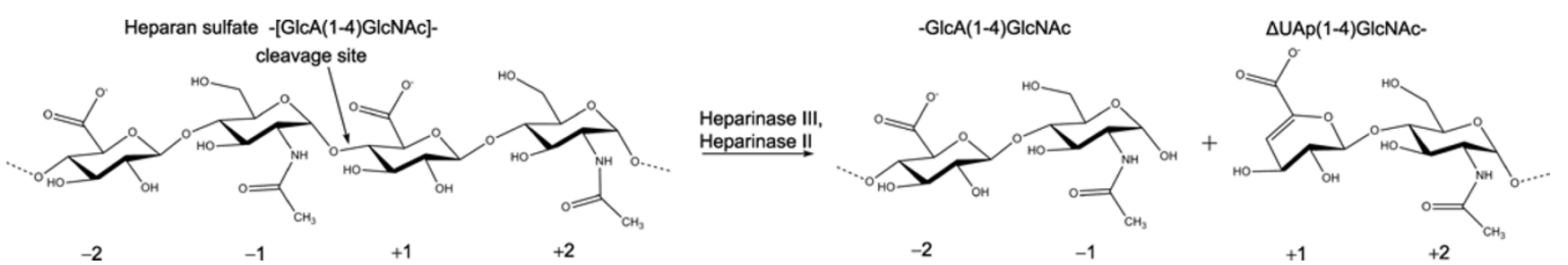

Scheme 1. Heparan sulfate degradation by heparinase III. The $\beta$-eliminative reaction for degradation of HS by both heparinase III and II is shown. The residue subsites are named according to the nomenclature by Davies et al (1997). 


\section{RESULTS}

\section{Substrate specificity and kinetic analysis}

Using a similar procedural method as for heparinase I (Luo et al., 2007), production of pure recombinant Heplll after affinity purification and GST-Tag removal is about $12 \mathrm{mg} / \mathrm{g}$ dry bacterial weight, which showed a single band with a molecular weight of about $73 \mathrm{kDa}$ (data not shown). Tests for different glycosaminoglycan substrates for degradative lyase activity of this purified Heplll revealed a high specificity for heparan sulfate (Fig. 1A), which is different from that reported for Bacteroides thetaiotaomicron heparinase I (Luo et al., 2007). No significant activity could be observed with heparin, chondroitin sulfate (Fig. 1A), dermatan or keratan sulfates (not shown). Examination of initial rates of Heplll activity over a
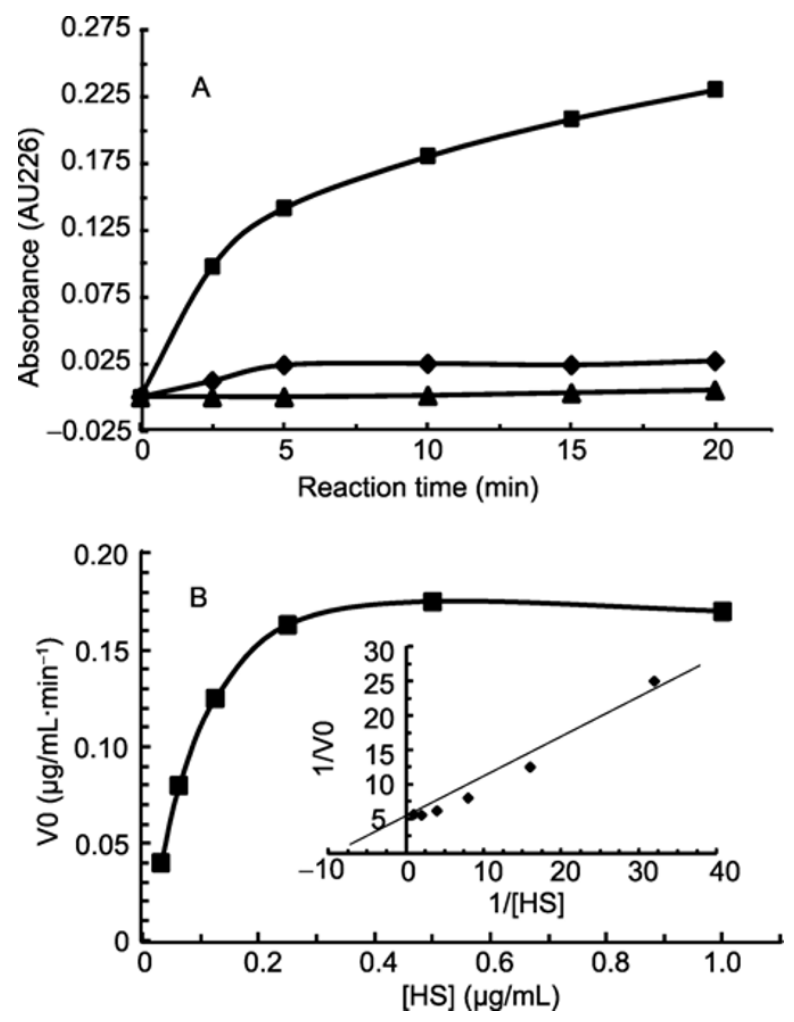

Figure 1. Substrate specificity and kinetic analysis of $B$. thetaiotaomicron heparinase III. (A) Heparan sulfate as enzymatic substrate. Comparison of purified Heplll lyase activity on bovine cartilage heparan sulfate (squares), porcine intestinal mucosa heparin (diamonds) and chondroitin sulfate (triangles) was done as described (Luo et al., 2007). (B) Kinetic analysis. The initial rates of $B$. thetaiotaomicron HepllI were determined in the presence of various concentrations of heparan sulfate as indicated. Data was fitted to the Michaelis-Menten equation and kinetic parameters were determined by nonlinear least-squares regression analysis. A double reciprocal plot of the data is also shown (inset). The indicated data is representative of three experiments with three independent Heplll preparations. range of $\mathrm{HS}$ substrate concentrations from 0.03125 to 1 $\mathrm{mg} / \mathrm{mL}$ incremented by a factor of 2 (2.6 to $83.3 \mu \mathrm{mol} / \mathrm{L}$ based on an average molecular mass of $12,000 \mathrm{Da}$ ) revealed a simple hyperbolic response curve. The calculated specific activity of the purified enzyme was 126 units/mg. Nonlinear least-squares regression analysis of the kinetic data by fitting to the Michealis-Menten equation indicated an apparent $\mathrm{Km}$ of $6.8 \pm 0.33 \mu \mathrm{mol} / \mathrm{L}$ (Fig. 1B). A double-reciprocal plot of the data conformed to a simple linear plot with a regression coefficient of 0.9895 (Fig. 1B, inset).

\section{Overall architecture of heparinase III}

To pinpoint its molecular mechanism underlying the substrate specificity, we determined the crystal structure of Heplll to an $R_{\text {free }}$ factor of $20.1 \%$ with data to $1.6 \AA$ resolution (Table 1 ). There exists one single Heplll molecule in an asymmetric unit. The model starts at Val44 and ends at Leu702, with an approximate size of $95 \times 50 \times 45 \AA$. The overall structure can be divided into two domains: an $\mathrm{N}$-terminal toroid-like domain (residue 44-408) and a C-terminal $\beta$-sandwich domain (residue 424-702) linked by a 15-residue loop (Fig. 2A). Both domains display distinguishable features compared to those of Hepll (Fig. 2B) (Shaya et al., 2006). In contrast, the $\mathrm{N}$-terminal portion of Hepl shrinks considerably, resulting in a $\beta$-jelly roll fold that is completely different from that of Hepll and Heplll (Han et al., 2009). Hence, we take the structure of Hepll as a significant reference for our study.

The $N$-terminal domain belongs to the $(\alpha / \alpha)_{5}$ toroid subclass, which starts with three small $\alpha$-helices ( $\alpha 1, \alpha 2$ and $\alpha 3$ ) and consists of $15 \alpha$-helices organized in a double-layered toroid fold (Fig. 2C). Helix $\alpha 1$ is short with only 6 residues. Helix $\alpha 2$ and $\alpha 3$ are arranged in an anti-parallel manner, whereas in Hepll, these two $\alpha$-helices are replaced by an anti-parallel $\beta$-strand pair (Fig. 2D). The core architecture is formed by five $\alpha$-helix hairpins ( $\alpha 6-\alpha 7, \alpha 8-\alpha 9, \alpha 10-\alpha 11$, $\alpha 12-\alpha 13, \alpha 14-\alpha 15)$. The outer layer consisting of $5 \alpha$-helices ( $\alpha 7, \alpha 9, \alpha 11, \alpha 13, \alpha 15)$ orients roughly towards the C-terminal domain. The inner layer ( $\alpha 6, \alpha 8, \alpha 10, \alpha 12, \alpha 14)$ possess a very similar arrangement to the outer shell in an opposite orientation. Compared to the $(\alpha / \alpha)_{6}$ toroid subclass of Hepll, the toroid of Heplll is incomplete with an $\alpha$-helix missing at the C-terminal of this domain. Hepll has an additional $\alpha$-helix ( $\alpha 14$, Fig. 2D and 3 ) forming the sixth $\alpha$-helix hairpins with its helix $\alpha 2$.

The C-terminal domain has a sandwich-shaped architecture consisting of $19 \beta$-strands forming three stacking $\beta$ sheets (Fig. 2E). Similar architecture is shared by all the toroid class members; however, this C-terminal domain in Hepll is twisted for approximately $60^{\circ}$ relative to the $\mathrm{N}$-terminal domain. The top layer proximal to the $\mathrm{N}$-terminal domain contains $8 \beta$-strands $(\beta 1-\beta 5, \beta 10, \beta 14-\beta 15)$ that are collocated in an anti-parallel arrangement. A very short $\alpha$-helix $(\mathrm{H} 1)$ inserted between strand $\beta 5 a$ and $\beta 5 b$ is perpendicular to the 
Table 1 X-ray data and refinement statistics

\begin{tabular}{|c|c|c|}
\hline Data set & Native & Se-Met (peak, max f") \\
\hline Wavelength $(\AA)$ & 0.9 & 0.9793 \\
\hline Space group & $\mathrm{C} 222_{1}$ & $\mathrm{C} 222_{1}$ \\
\hline \multirow[t]{3}{*}{ Unit cell } & $a=168.7 \AA$ & $a=171.3 \AA$ \\
\hline & $b=210.0 \AA$ & $b=210.2 \AA$ \\
\hline & $c=84.9 \AA$ & $c=85.2 \AA$ \\
\hline Resolution ( $(\AA)$ & 1.6 & 2.05 \\
\hline Measured reflections & $3,321,831$ & 520,293 \\
\hline Unique reflections & 193,324 & 93,790 \\
\hline Redundancy & 17.2 & 5.5 \\
\hline Completeness (\%, highest shell) & $97.7(93.4)$ & $97.4(93.8)$ \\
\hline Mean I/бI (highest shell) & $16.7(3.6)$ & $7.6(1.2)$ \\
\hline Rsym (\%, highest shell) & $10.7(42.4)$ & $17.3(79.3)$ \\
\hline \multicolumn{3}{|l|}{ Refinement } \\
\hline Resolution $(\AA)$ & 1.6 & \\
\hline No. of reflections $|\mathrm{F}|>0 \sigma \mathrm{F}$ & 189,008 & \\
\hline R-factor/R-free (\%) & $17.9 / 19.8$ & \\
\hline No. of protein atoms & 5283 & \\
\hline No. of water molecules & 1390 & \\
\hline rmsd bond lengths $(\AA)$ & 0.008 & \\
\hline rmsd bond angles $\left({ }^{\circ}\right)$ & 1.149 & \\
\hline
\end{tabular}

sheet plane, as a landmark dividing this $\beta$ sheet into two parts. The middle layer has a similar anti-parallel arrangement containing $8 \beta$-strands ( $\beta 6-\beta 9, \beta 11-\beta 13, \beta 16)$; whereas the bottom layer is narrow containing only three anti-parallel $\beta$-strands $(\beta 17-\beta 19)$.

$A$ unique feature of Heplll is a tunnel formed between the two domains and a loop (Fig. 2A, 2C and 2E). This loop (L1) between $\alpha 4$ and $\alpha 5$ contains 38 residues (Figs. 2C and 3), whereas the corresponding one in Hepll has only 13 residues (Figs. 2D and 3). This loop coils up and contacts two stretching out loops, L2 (between $\beta 5 a-\beta 5 b)$ and L3 (between $\beta 14-\beta 15)$ from the C-terminal domain (Fig. 2E). These loops together form a wall that covers the entrance of the cleft between two domains, turning the cleft into a tunnel.

\section{Potential substrate binding site with a bound HS tetrasaccharide}

Both HepllI and Hepll degrade HS, share an approximately $25 \%$ sequence similarity and a similar overall architecture, suggesting that they might share a similar substrate binding site. Structure-based sequence alignment showed that the crucial residues in Hepll related to degrading reaction are well-conserved in Heplll (Fig. 3) (Shaya et al., 2006, 2010). These important residues include Tyr314 and His464 in HepIII, and Tyr257 and His406 in Hepll. Based on the residue conservation in the primary sequence and the superimposition of the conserved catalytic residues (Fig. 3 and 4), we modeled a tetrasaccharide HS motif, $\triangle U A(1-4) G I c N A c(1-4)$ GlcA(1-4)GlcNAc, as a bound substrate in the tunnel, where the potential active site with conserved catalytic residues are located (Fig. 4A). The modeled substrate fits in the active site and adopts an extended conformation at a low energy state.

The tunnel at the interface of the two domains is $25 \AA$ long with a large entrance at one end $(\sim 25 \times 15 \AA)$ and a relatively smaller entrance $(\sim 20 \times 10 \AA)$ at the other end (Fig. 4B). The middle part connecting the two ends is constricted to a small hole, dividing the tunnel into two parts. The part at the large entrance forms a cavity which accommodate the "-subsites" of the substrate, while the other part at the smaller entrance is occupied by the "+subsites" (Scheme 1). The tunnel is composed almost entirely of residues from the inner shell of the toroid ( $\alpha 4, \alpha 5, \alpha 6, \alpha 8, \alpha 10, \alpha 12, \alpha 14$ and loops between $\alpha 3-\alpha 4, \alpha 4-\alpha 5, \alpha 13-\alpha 14)$ and the top layer of the C-terminal domain (loops between $\beta 3-\beta 4, \beta 14-\beta 15)$. The inner surface of the tunnel in Heplll is only partially with a positive potential (Fig. 4A), consistent with its substrate specificity, as will be discussed later.

The tetrasaccharide adopts a linear extension form with the non-reducing end toward the large entrance end and the reducing end toward the smaller entrance end (Fig. 4B). The -2 and +1 subsites are occupied by GlcA, and the -1 and +2 
A

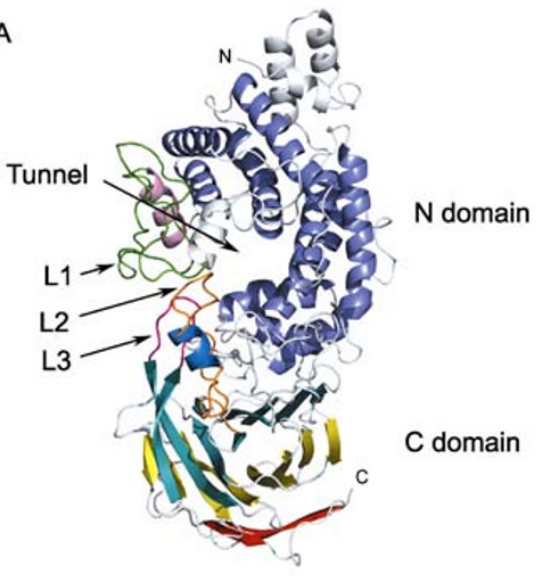

C

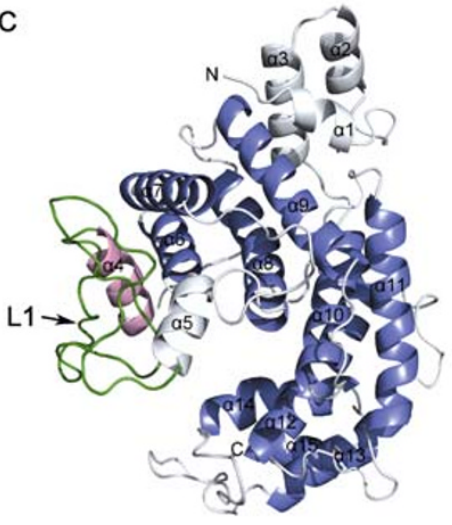

$\mathrm{E}$

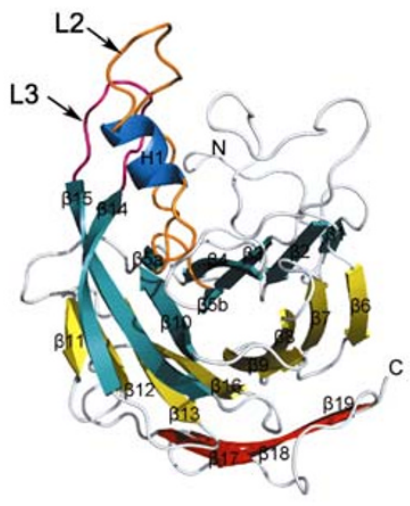

B

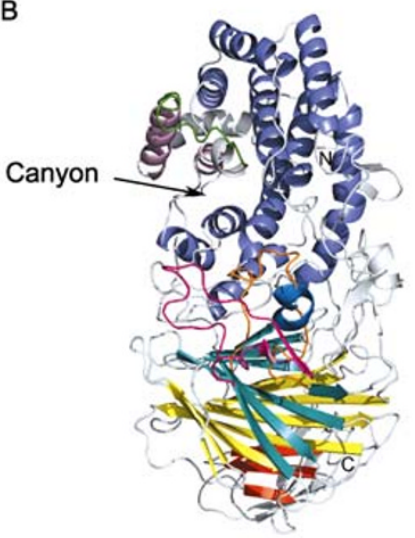

D
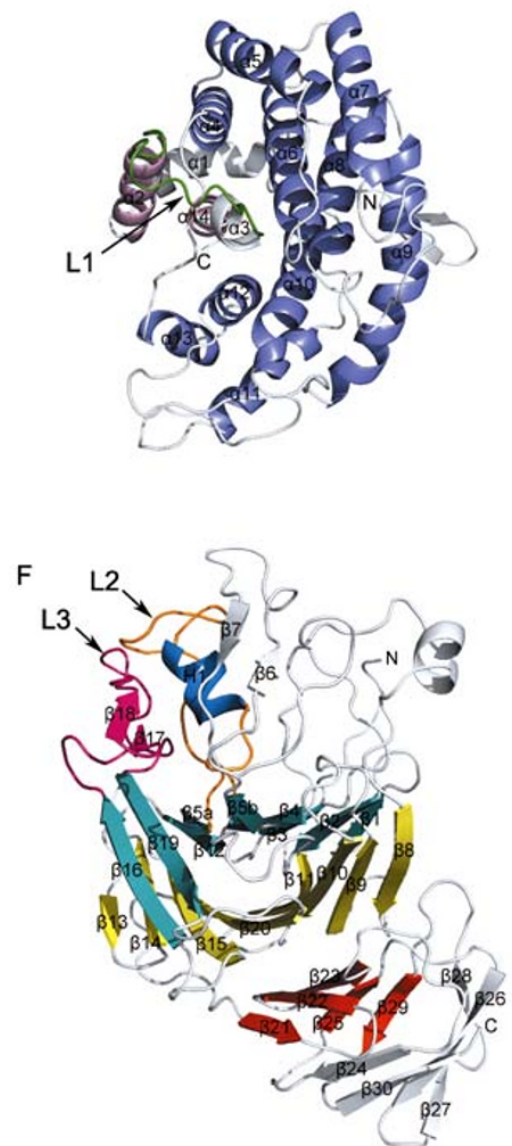

Figure 2. Comparative representation of the structures for heparinase III and heparinase II. (A) Schematic representation of overall structure of heparinase III with an $\alpha$-helices toroid $\mathrm{N}$-terminal domain and a $\beta$-sandwich $\mathrm{C}$-terminal domain; (B) Comparative overall structure of heparinase II; (C) The N-terminal domain of Heplll with the $(\alpha / \alpha)_{5}$ toroid colored blue. Secondary structure elements are marked, the Loop L1 is colored green and indicated by arrow; helix $\alpha 4$ is colored pink. (D) The N-terminal domain of Hepll with the $(\alpha / \alpha)_{6}$ toroid colored as in panel $C$, the additional helix pair is colored pink. Secondary structures are marked. The helices $\alpha 2$ and $\alpha 14$ comprising an additional helix hairpin of Hepll are colored pink as in panel C; (E) The C-terminal domain of heparinase III with the first layer colored deepteal, the middle layer colored yellow and the bottom layer colored red. Secondary structure elements are marked, the loop L2 and L3 are colored orange and hotpink respectively, and both are indicated by arrows; (F) The central domain and part of the C-terminal domain of Hepll, that are corresponding to the C-terminal domain of HepllI, are rotated for approximately 60 about the vertical axis for clarification and are colored as in (E). This figure was prepared with PyMol. 
A

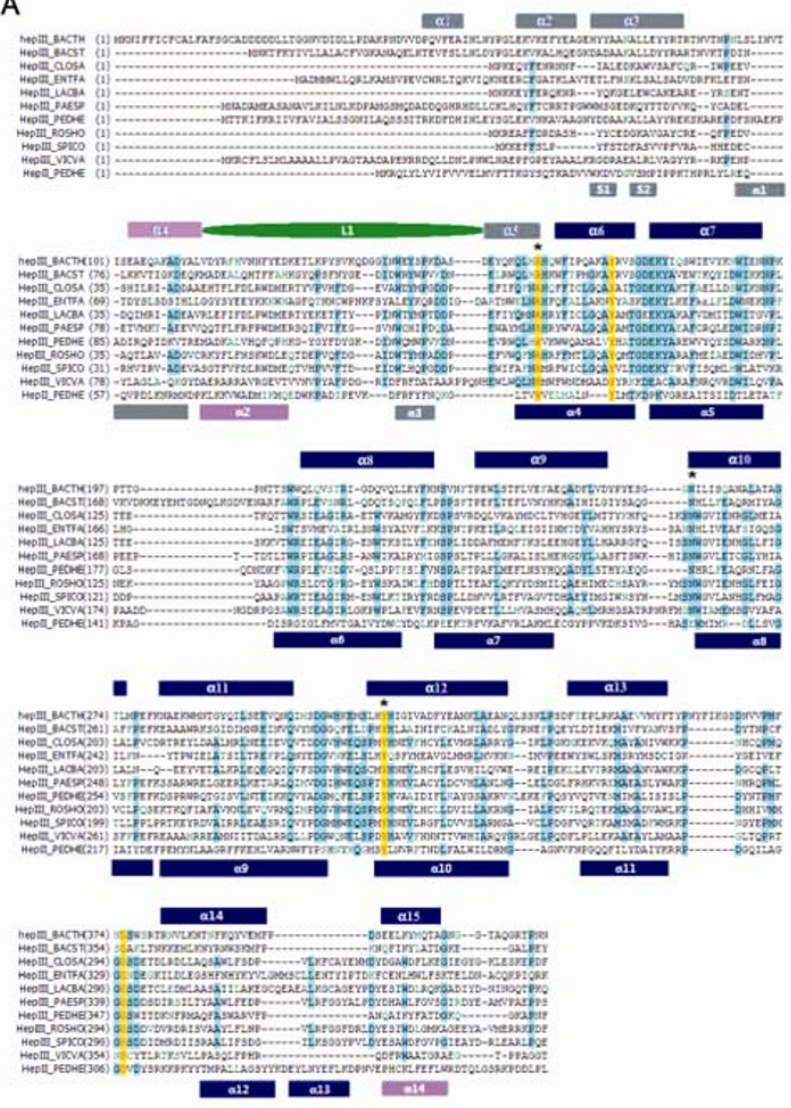

B
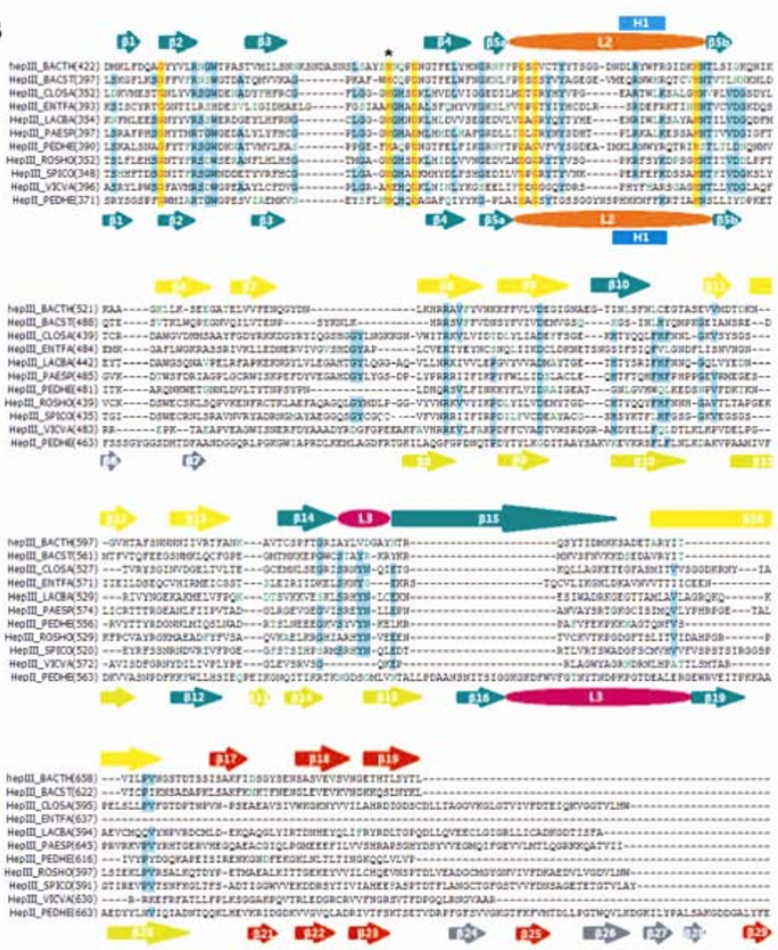

Figure 3. Sequence alignment of Heplll from 12 bacteria and Hepll from Pedobacter heparinus. The sequences of heprinases III are from the following species: BACTH, Bacteroides thetaiotaomicron; BACST, Bacteroides stercoris; CLOSA, Clostridium saccharolyticum; ENTFA, Enterococcus faecium; LACBA, Lachnospiraceae bacterium; PAESP, Paenibacilus sp; PEDHE, Pedobacter heparinus; ROSHO, Roseburia hominis; SPICO, Spirochaeta coccoides; VICVA, Victivallis vadensis. The sequence of heprinases II is from PEDHE, Pedobacter heparinus. The secondary structures of Heplll and Hepll based on the crystal structure are shown above and below the sequence alignment respectively, and colored as in Fig. 1. The $\alpha$ helices and the $\beta$ strands are shown as boxes and arrows respectively. (A) Sequence alignment of the N-terminal domain of HepllI and Hepll. The $\alpha$ helices belonging to the toroid are colored in blue; $(\mathrm{B})$ Sequence alignment of the C-terminal domain of Heplll and the middle domain along with the C-terminal domain of Hepll. is colored blue. The first layer of the C-terminal domain is colored deepteal, the middle layer in yellow and the bottom layer in red. Loops L1, L2 and L3 are shown as green, orange and purple oval, respectively. The highly conservative residues related to catalytic reaction are marked with an asterisk above the sequence.

subsites are filled with GlcNAc. The two sugar rings of the disaccharide unit at the +1 and +2 subsites appear almost coplanar with each other, while the two at the minus subsites are bent at the glycosidic bond between the plus and minus subsites.

The tetrasaccharide bound in the tunnel intimately contacts multiple side chains (Fig. $4 \mathrm{C}$ and Table 2). The substrate is stabilized mainly by hydrogen bonds (Table 2, Fig. $4 C$ ). The carboxylic group of the GlcA residues and most of the hydroxyl group are likely bound to the side chains of residues from the inner surface of the tunnel. The glucosamine lying at the +2 subsite maintains its position by two hydrogen bonds, one between the 2-acetyl group of GIcNAc and the hydroxyl group of Tyr255, and the other one between the 6-hydroxyl group of GlcNAc and the terminal amino group of Lys157. The GlcA at the +1 subsite is most likely involved in the cleavage reaction. Its sugar ring is possibly twisted due to the strong interactions surrounding it, and hence adopts a non-canonical boat conformation. This residue is firmly held by several hydrogen bonds. The two carboxylic oxygen atoms of GlcA at the +1 subsite form two hydrogen bonds with the amide group of Asn260. Due to the limited space, the two hydrogen bonds are short, indicating strong interactions. On the other side of the queue of the sugar rings, three short hydrogen bonds form with the conserved Gly153 and Arg160, well positioning the sugar ring for reaction (Fig. 4C). The C-5 atom, one of the crucial atoms in elimination reaction, stays close to the hydroxyl group of Tyr314 with a relatively short 
Table 2 Hydrogen bonds between the modeled tetrasaccharide substrate and heparinase III

\begin{tabular}{lll}
\hline \multicolumn{2}{c}{ Tetrasaccharide } & Heplll \\
\cline { 1 - 2 } Subsite number & Atom & ND1-His313 \\
\hline-2 & O2 & O-His313 \\
-1 & O2 & OG-Ser264 \\
& O6 & NH1-Arg160 \\
& O7 & NE2-Gln157 \\
& O7 & OE2-Glu153 \\
+1 & N2 & OE1-Glu153 \\
& O2 & NH2-Arg160 \\
& O3 & OE1-Glu153 \\
& O3 & NE2-His464 \\
& O5 & ND2-Asn260 \\
& O6A & OD1-Asn260 \\
& O6B & NZ-Lys156 \\
& O6 & OH-Tyr255 \\
& O7 & OH-Tyr314 \\
\hline
\end{tabular}

distance, which allows the occurrence of the C-5 proton abstraction. The $\mathrm{O} 4$ atom of the glycosidic bond is another key atom involved in the reaction. It could interact with the hydroxyl group of Tyr314 through a strong hydrogen bond in light of their close positions. In general, the minus subsites possibly make fewer interactions with the protein residues compared with the plus subsites. The amide group of GIcNAc at the -1 subsite is held by three hydrogen bonds. The N2 atom interacts with the carboxylic group of Glu153, whereas the 07 binds to the guanidinium group of Arg160 and the amide group of $\mathrm{G} \ln 157$. On the other side of the sugar ring, the $\mathrm{O} 6$ atom also contributes to the stabilization by interacting with the hydroxyl group of Ser264. The interaction between the GlcA at the -2 subsite residue is relatively weak and only two hydrogen bonds are formed. The $\mathrm{O} 2$ atom plays a part in the stabilization by making two hydrogen bonds to the mainchain carbonyl oxygen atom of His313 and its side chain imidazole group.

\section{DISCUSSION}

Based on the primary sequence, Heplll can be assigned to the PL12 family (Cantarel et al., 2009). To date, most of the 21 members of the PL families have been structurally well studied, except the PL12 and PL17. Our study provides a representative structure of the PL12 family. Heparinase III shares a similar overall structure to heparinase II and Chondroitinase AC (ChonAC) which have been structurally well-characterized (Lunin et al., 2004; Shaya et al., 2006). These three proteins share a similar architecture composed of an $(\alpha / \alpha)_{5,6}$ toroid and $\beta$-sandwich fold. The toroid of HepllI is incomplete compared to that of Hep II. Helices $\alpha 2$ and $\alpha 14$ of Hepll, which are distant in the primary sequence, form an a-helix pair to hold the ends together (Fig. 2D). However, in HepllI, this pair does not exist due to the missing of an $\alpha$ helix at the C-terminal end of the toroid, resulting in the formation of an open entrance with a approximate width of $15 \AA$ (Fig. 2C). This character is observed in ChonAC that also adopts a $(\alpha / \alpha)_{5}$ fold at the $\mathrm{N}$-terminus. The $\mathrm{C}$-terminal domain of HepllI is organized into a triple-sheet $\beta$ sandwich whereas the corresponding parts of both Hepll and Chondroitinase AC are assembled into a four antiparallel $\beta$-sheets (Fig. 2E and 2F). This difference is resulted mainly from the lack of several $\beta$ strands at the most C-terminal end of Heplll, and makes the C-terminal domain of Heplll a downsized counterpart of Hepll.

The potential substrate binding site of Heplll is different from that of Hepll in several aspects. Firstly, the overall structure of substrate binding site of Heplll is located in a tunnel, while that of Hepll is in a canyon. Secondly, the electrostatic potential of the site of HepllI is much lower than that of Hepll. Thirdly, the most obvious difference is that helix $\alpha 5$ and the loop connecting $\alpha 4$ and $a 5$ are much more close to the C-terminal domain in Heplll. This segment is located at a position similar to the cleft entrance in Hepll, and converts the canyon in Hepll into a tunnel by standing as a wall. Some residues of the substrate binding site display a high degree of conservation. Residues Tyr314, His464, Asn260 and Arg160 of Heplll can be well superimposed with Tyr257, His406, Glu205 and Asp145 of Hepll (Fig. 4D), and the first three of them are directly related to the active site, respectively.

A distinguishing characteristic of Heplll is its ability to specifically cleave the oxygen-aglycone at sulfate-rare region in HS, but not heparin (Linhardt et al., 1990). Two factors probably contribute to this specificity. Firstly, the inner surface of the tunnel in Heplll seems to be less positively charged than that in Hepl (Han et al., 2009), consistent with the lower sulfation thus less negative charge of HS compared to heparin (Fig. 4A). Among a variety of modification patterns of heparin and $\mathrm{HS}$, the sulfation at 2-O of UA is considered to be the most critical one to determine the substrate specificity. 2-O-unsubstitution of GIcA and IdoA is a prerequisite for Heplll to degrade HS whereas 2-O-sulfation of IdoA is required for heparin degradation. In our model, the 2-O of the GlcA residue at the +1 subsite is located quite close to the $\mathrm{N}$-terminus of helix $\alpha 5$, and the space between them is too limited to accommodate an extra sulfate group modifying the 2-O atom. Thus, heparin that is highly sulfated at 2-O of IdoA and GIcA are probably spatially refused to enter the substrate binding site of HepllI.

Based on the model presented here, we propose a putative mechanism to explain the exclusive elimination reaction of HepllI (Fig. 5). The catalytic reaction includes three main steps: (1) neutralization of negative charge of the GlcA carboxylic group to reduce the pKa of C-5 proton; (2) abstraction of the C- 5 proton by a general base; (3) donation of a proton by a general acid to produce a normal hydroxyl group at the reducing end of the product. 
A

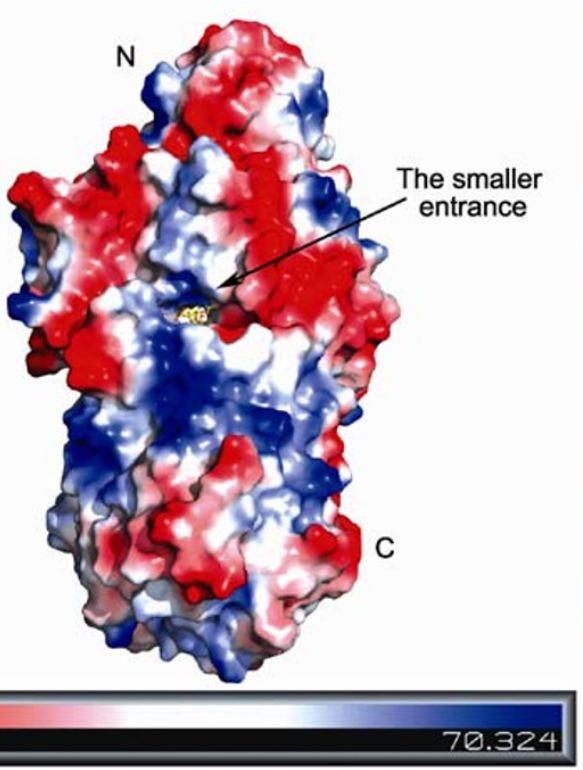

C

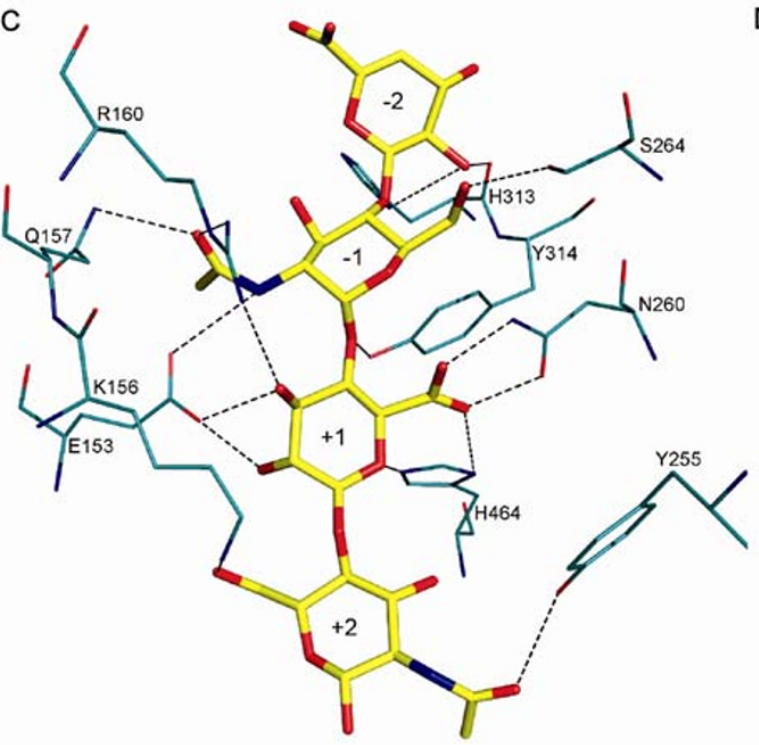

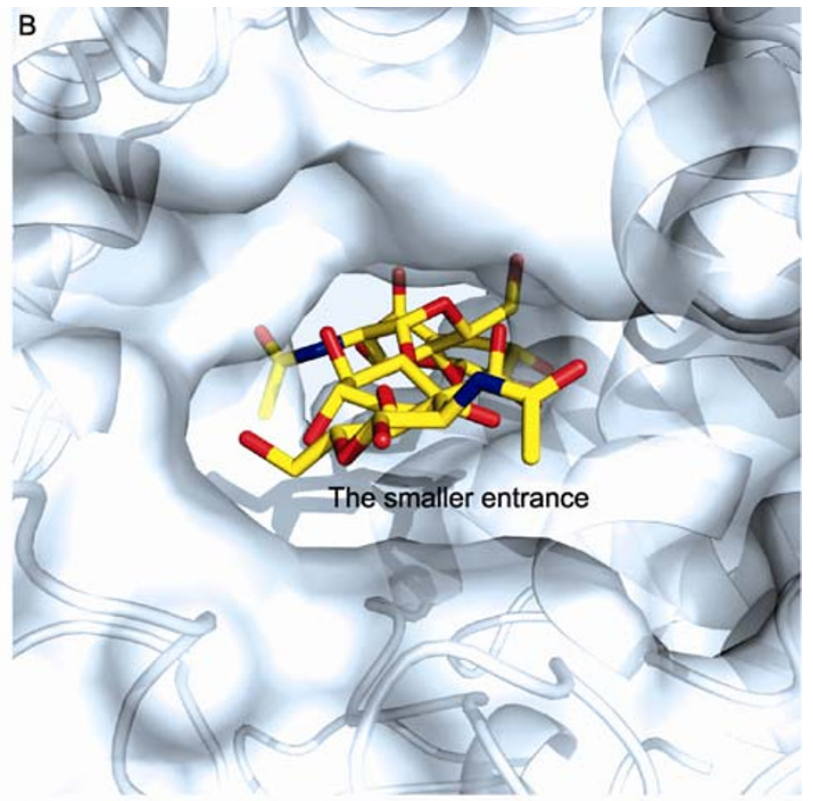

D

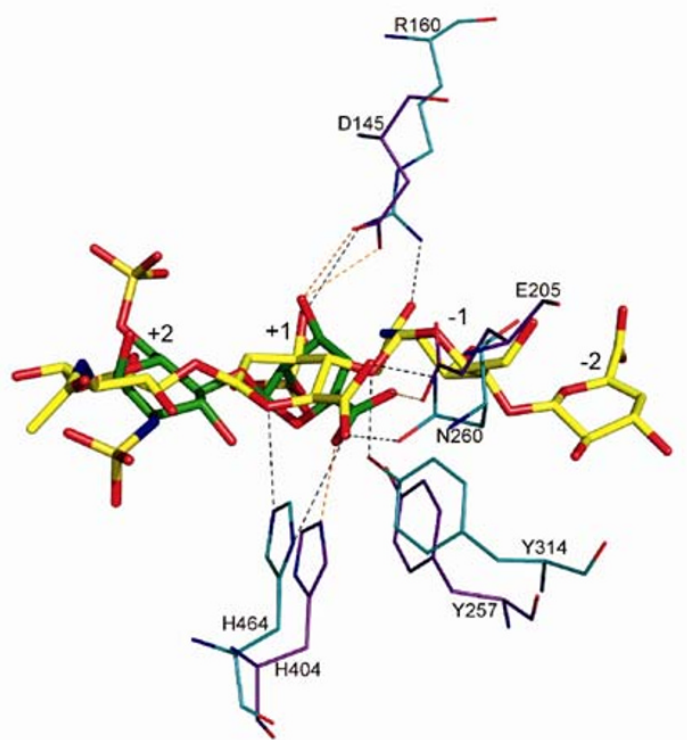

Figure 4. The substrate binding site of heparinase III. (A) Surface charge presentation of the heparinase III structure with a bound tetrasaccharide HS modeled based on Hep II structure. (B) The close-up view of the tunnel in heparinase III with a modeled tetrasaccharide substrate. The tetrasaccharide is shown in sticks and its backbone is colored yellow, with the minus subsites facing outward. (C) Molecular details of the structure and interactions within the substrate binding site of heparinase III. The modeled HS tetrasaccharide is shown as in $\mathrm{B}$, conservative residues related to the binding site are shown in thin lines and colored deepteal, the hydrogen bonds are shown in black dash lines. (D) Superimposition of the active site of heparinse III and heparinase II based on the conservative catalytic residues. The modeled tetrasaccharide in Heplll and the hydrogen bonds between the substrate and the related residues are shown as in C. The disaccharide product of Hepll is shown in sticks and colored forest, and the related key residues for catalysis are shown in thin lines and colored purple. The hydrogen bonds between the product and related residues are shown as orange dashes.

Two different approaches are employed to neutralize the negative charge on the carboxylic group. Pectin lyase utilizes a $\mathrm{Ca}^{2+}$ ion to weaken the negative charge (Mayans et al., 1997) while others like Hepll (Shaya et al., 2006) and Cho-
nAC (Lunin et al., 2004) exert their asparagine residue to achieve the process. In our model, Heplll likely implements a similar strategy as ChonAC and Hepll to accomplish the neutralization by employing its residue Asn260. The side 


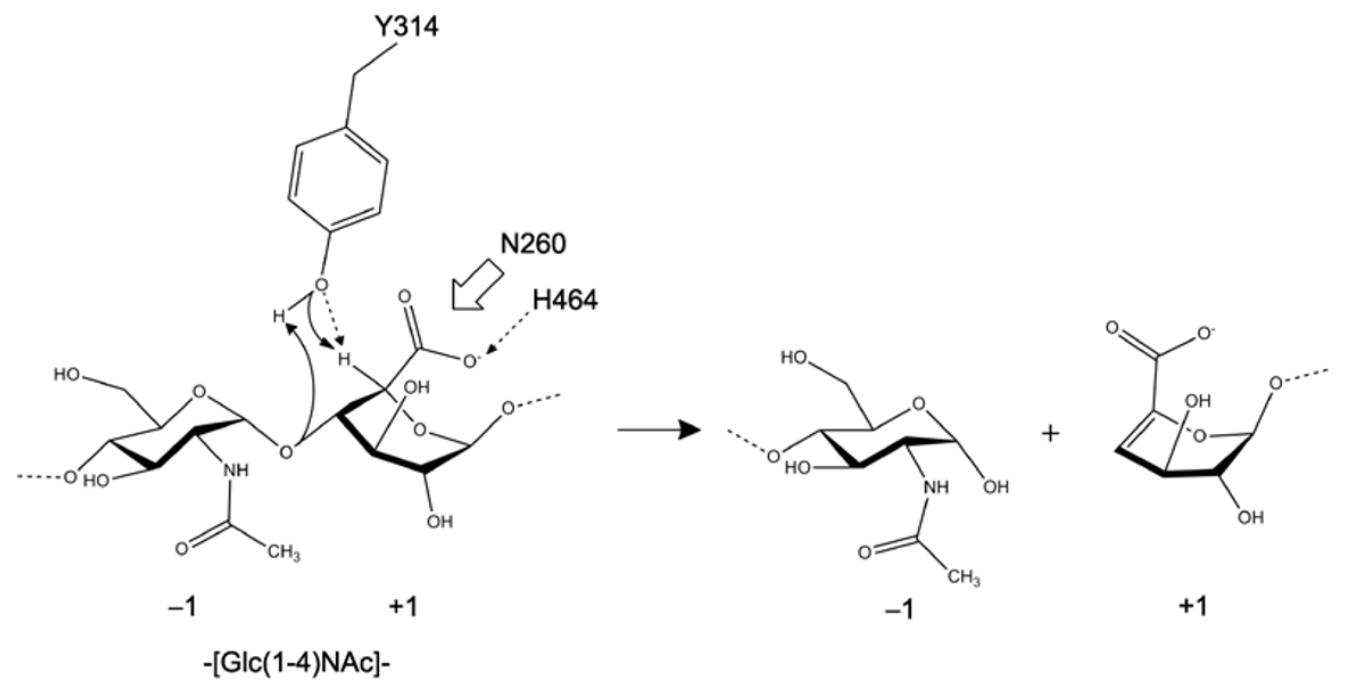

Figure 5. Schematic representation of the proposed catalytic mechanism. The N260 and H464 residues in Heplll likely contribute to the neutralization of carboxylic group, and Y 314 is considered to play a critical dual-function role both in the abstraction of the $\mathrm{C}-5$ proton and donation of a proton to reconstitute the hydroxyl group at the reducing end of the product.

chain of Asn260 of Heplll is very close to the carboxylic group of GlcA at the +1 subsite. The distance between the ND2 and O6A atom and that between the OD1 and the O6B are in a proper range to create tight hydrophilic interactions. Such a short distance could probably leads to hydrogen bond formation as long as the carboxylic group is in a protonated state. Subsequently, the neutralization results in a large reduction of the pKa of the C-5 proton. This neutralization manner of HepIII is very similar to that of ChonAC lyase which engages its N183 in an equivalent position to serve in the same function. While in Hepll, three residues E205, R261 and H404 combine to take the responsibility for neutralization. In addition, the NE of His464 in Heplll is positioned to the carboxylic group laterally with a distance in a hydrogen-forming range, indicating it may also contribute to the neutralization. The abstraction of the C-5 proton is probably accomplished by highly conserved Tyr314, considering its vicinity position to the C-5 atom. Its counterpart in Hepll is the Tyr257 residue that is located to a similar position and has been proved to serve both as a general acid and a general base. In Heplll, the distance between the hydroxyl group of Tyr314 and the $\mathrm{C}-5$ atom is within a strong hydrogen bond range, which provides a reasonable environment and a possibility for the hydroxyl oxygen atom to abstract the proton from the C-5 atom. Besides serving as a general base in the proton abstraction step, Tyr314 probably also plays a critical role in proton donation. Tyr314 is very close to the $\mathrm{O} 4$ atom of the glycosidic bond that is the accepter of the proton. In the final step, a proton is donated by the hydroxyl group of Tyr314 to the O4 atom of the glycosidic bond to produce a new hydroxyl group at the reducing end of the product. The glycosidic bond is broken at the same time of the proton acceptance by the $\mathrm{O} 4$ atom, coupled with the formation of a double bond between $\mathrm{C}-4$ and $\mathrm{C}-5$ atoms at the non-reducing end. Hence, the
H464 residue probably contributes to the neutralization, and Tyr314 obviously plays a dual-function role in the elimination reaction both as a general base and a general acid.

In summary, we present here the crystal structure, modeling and biochemical analyses of Heplll that is an HS-specific degrading PL. The structure clearly shows that it belongs to the $(\alpha / \alpha)_{5}$ toroid subclass with a toroid-like $\mathrm{N}$-terminal domain and a $\beta$-sandwich $\mathrm{C}$-terminal domain. Furthermore, we built a model with a tetrasaccharide HS substrate bound to the Heplll enzyme, which possibly provides us a deep insight into its catalytic mechanism and specificity for degradation of poor-sulfated HS but not highly sulfated heparin motif. The potential active site is placed at a tunnel where the $\mathrm{N}$-terminal domain is more involved. The enzyme may probably employ a similar approach as ChonAC and Hepll to accomplish the reaction by engaging the major conserved Tyr314 as a dual-function active residue.

\section{MATERIAL AND METHODS}

\section{Materials}

B. thetaiotaomicron was from the American Type Culture Collection (Manassas, VA). Heparin (porcine intestinal mucosa, 170 USP units/mg), heparan sulfate (bovine kidney), chondroitin sulfate (bovine cartilage), dithiothreitol (DTT) and reduced glutathione were from Sigma (St. Louis, MO). Thrombin, HiTrap Glutathione-Sepharose, Benzamidine-Sepharose and Superose 12 columns were from GE HealthCare Bio-Sciences Corp (Piscataway, NJ).

\section{Protein expression, purification, and crystallization}

Culture of $B$. thetaiotaomicron and the recovery of bacterial genomic DNA were described as a previous report (Luo et al., 2007). DNA 
encoding $B$. Thetaiotaomicron heparinase III (cDNA, AAO79767) was prepared using PCR with primers 5'-GGGGATCCATGCTGA CTGCTCAGACT-3' and 5'-GGGAATTCTTATCTTTCCGAATATCC-3' and purified genomic DNA as template. The PCR product was digested and ligated into pGEX-2T vector at BamHI and EcoRI sites in frame with coding sequence for GST at the $5^{\prime}$ end. A thrombin/trypsin cut site was introduced between the GST and heparinase III. The expression of GST-heparinase III and purification of GST-heparinase III and heparinase III followed a similar procedural method as described (Luo et al., 2007).

\section{Crystallization}

Heparinase III free of GST fusion was subjected to crystallization screening under a wide range of conditions. Sitting/hanging drop vapor diffusion method was applied in crystallization. The protein was mixed with reservoir solution at a ratio of $1: 1$. The well-diffracting crystals were grown by handing drop vapor diffusion where $2 \mu \mathrm{L}$ of the Heplll protein solution at a concentration of $10 \mathrm{mg} / \mathrm{mL}$ was mixed with an equal volume of crystallization solution containing $3 \mathrm{~mol} / \mathrm{L}$ Sodium Formate and $0.1 \mathrm{~mol} / \mathrm{L}$ Tris- $\mathrm{HCl}, \mathrm{pH} 8.0$.

\section{Data collection and structure determination}

Crystals were cryo-protected from their mother liquid containing $30 \%$ glycerol, and were frozen in liquid nitrogen. A native data set of Heplll and a single-wavelength anomalous diffraction (SAD) data set of selenomethionine Heplll were collected to $1.6 \AA$ and $2.05 \AA$ resolutions, each from a single crystal at the Shanghai Synchrotron Radiation Facility (SSRF) BL17U beamline (Shanghai, China). The data were indexed, integrated and scaled using HKL2000 (Otwinowski et al., 1997). Data collection statistics were summarized in Table 1. Crystals are in space group $\mathrm{C} 222_{1}$ with one molecule in an asymmetric unit. The crystal structure was solved by SAD, Thirteen selenium sites in the asymmetric unit were found by using SHELXD (Schneider and Sheldrick, 2002). Final refinement of selenium parameters and calculation of phases were performed with the program MLPHARE (CCP4, 1994), giving a readily interpretable electron density map. The model building was carried out with the program COOT (Emsley and Cowtan, 2004) and subsequent refinement using REFMAC5 (Murshudov et al., 1997).

\section{Heparinase III enzyme kinetics and specificity}

Heparinase III activity was determined by ability to cleave heparan sulfate that yields an unsaturated double bond at C4-C5 position at the non-reducing terminus of the resulting saccharide with a peak absorbance at about $230 \mathrm{~nm}$ ( 226 was used in the current study). The initial reaction rate in kinetic analyses of heparinase III enzyme was determined by the increase in absorbance for the first $1 \mathrm{~min}$ at $37^{\circ} \mathrm{C}$ with $\mathrm{HS}$ concentrations ranging from $0.03125 \mathrm{mg} / \mathrm{mL}$ to 1 $\mathrm{mg} / \mathrm{mL}$ and $1 \mu \mathrm{g} / \mathrm{mL}$ heparinase III in $1 \mathrm{~mL}$ buffer containing 20 $\mathrm{mmol} / \mathrm{L}$ Tris- $\mathrm{HCl}, \mathrm{pH} 7.0,0.15 \mathrm{~mol} / \mathrm{L} \mathrm{NaCl}, 1 \mathrm{mmol} / \mathrm{L} \mathrm{CaCl} 2$. Unless otherwise noted, all enzyme assays consisted of buffer $\mathrm{B}$ containing $1 \mathrm{mmol} / \mathrm{L}$ freshly prepared DTT. Enzymatic activity, kinetic parameters and substrate specificity were determined as described for hepa- heparinase I (Luo et al., 2007). In specificity analyses, $0.2 \mathrm{mg} / \mathrm{mL}$ heparin, HS or CS were used together with $2 \mu \mathrm{g} / \mathrm{mL}$ heparinase III. Analyses were performed at least three times with independent preparations of heparinase III. Unless otherwise indicated, a single representative experiment showing the mean of triplicate assays \pm $\mathrm{SD}$ is presented in the text.

\section{ACKNOWLEDGMENTS}

We thank Sheng Huang and Jianhua $\mathrm{He}$ at Shanghai Synchrotron Radiation Facility (SSRF) for on-site assistance. This work was supported in part by funds from Ministry of Science and Technology (No. 2011CB910500), the National Natural Science Foundation of China (Grant No. 31070661), the Natural Science Foundation of Zhejiang Province (No. R2100439), the Specialized Research Fund for the Doctoral Program of Higher Education (No. 20110101110122), and the Fundamental Research Funds for the Central Universities (SY), US Public Health Service grants (No. DK56338) (Texas Medical Center Digestive Diseases Center) and Texas A\&M Health Science Center Enhancement Grant (YL), and CA05997 and P50 CA140388 (WLM). The structure coordinates and reflection files are deposited in the protein data bank under accession number 4FNV.

\section{ABBREVIATIONS}

FGF, fibroblast growth factor; FGFR, FGF receptor; FPLC, flow perfromance liquid chromatography; GAG, glycosaminoglycan; GlcA, glucuronic acid; GlcNAc, N-acetylated glucosamine; GlcNS, $\mathrm{N}$-sufated glucosamine; Hep I, heparinase 1; Hepll, heparinase II; HepIII, heparinase III; HS, heparan sulfate; IdoA, iduronic acid; PL, polysaccharide lyase; UA, uronic acid

\section{REFERENCES}

Bornemann, D.J., Duncan, J.E., Staatz, W., Selleck, S., and Warrior, R. (2004). Abrogation of heparan sulfate synthesis in Drosophila disrupts the Wingless, Hedgehog and Decapentaplegic signaling pathways. Development 131, 1927-1938.

Bulow, H.E., and Hobert, O. (2006). The molecular diversity of glycosaminoglycans shapes animal development. Annu Rev Cell Dev Biol 22, 375-407.

Cantarel, B.L., Coutinho, P.M., Rancurel, C., Bernard, T., Lombard, V., and Henrissat, B. (2009). The Carbohydrate-Active EnZymes database (CAZy): an expert resource for Glycogenomics. Nucleic Acids Res 37, D233-238.

Capila, I., and Linhardt, R.J. (2002). Heparin-protein interactions. Angew Chem Int Ed Engl 41, 391-412.

CCP4 (1994). The CCP4 suite: programs for protein crystallography. Acta Crystallogr D Biol Crystallogr 50, 760-763.

Davies, G.J., Wilson, K.S., and Henrissat, B. (1997). Nomenclature for sugar-binding subsites in glycosyl hydrolases. Biochem J 321 (Pt 2), 557-559.

Desai, U.R., Wang, H.M., and Linhardt, R.J. (1993a). Specificity studies on the heparin lyases from Flavobacterium heparinum. Biochemistry 32, 8140-8145.

Desai, U.R., Wang, H.M., and Linhardt, R.J. (1993b). Substrate 
specificity of the heparin lyases from Flavobacterium heparinum. Arch Biochem Biophys 306, 461-468.

Emsley, P., and Cowtan, K. (2004). Coot: model-building tools for molecular graphics. Acta Crystallogr D Biol Crystallogr 60, 2126-2132.

Ernst, S., Langer, R., Cooney, C.L., and Sasisekharan, R. (1995). Enzymatic degradation of glycosaminoglycans. Crit Rev Biochem Mol Biol 30, 387-444.

Esko, J.D., and Selleck, S.B. (2002). Order out of chaos: assembly of ligand binding sites in heparan sulfate. Annu Rev Biochem 71, 435-471.

Godavarti, R., and Sasisekharan, R. (1996). A comparative analysis of the primary sequences and characteristics of heparinases I, II, and III from Flavobacterium heparinum. Biochem Biophys Res Commun 229, 770-777.

Guimond, S.E., and Turnbull, J.E. (1999). Fibroblast growth factor receptor signalling is dictated by specific heparan sulphate saccharides. Curr Biol 9, 1343-1346.

Han, C., Belenkaya, T.Y., Khodoun, M., Tauchi, M., and Lin, X. (2004). Distinct and collaborative roles of Drosophila EXT family proteins in morphogen signalling and gradient formation. Development 131, 1563-1575.

Han, Y.H., Garron, M.L., Kim, H.Y., Kim, W.S., Zhang, Z., Ryu, K.S., Shaya, D., Xiao, Z., Cheong, C., Kim, Y.S., et al. (2009). Structural snapshots of heparin depolymerization by heparin lyase I. J Biol Chem 284, 34019-34027.

Harmer, N.J. (2006). Insights into the role of heparan sulphate in fibroblast growth factor signalling. Biochem Soc Trans 34, 442-445.

Jackson, R.L., Busch, S.J., and Cardin, A.D. (1991). Glycosaminoglycans: molecular properties, protein interactions, and role in physiological processes. Physiol Rev 71, 481-539.

Kamimura, K., Koyama, T., Habuchi, H., Ueda, R., Masu, M., Kimata, K., and Nakato, H. (2006). Specific and flexible roles of heparan sulfate modifications in Drosophila FGF signaling. J Cell Biol 174, 773-778.

Kan, M., Wang, F., Xu, J., Crabb, J.W., Hou, J., and McKeehan, W.L. (1993). An essential heparin-binding domain in the fibroblast growth factor receptor kinase. Science 259, 1918-1921.

Kan, M., Wu, X., Wang, F., and McKeehan, W.L. (1999). Specificity for fibroblast growth factors determined by heparan sulfate in a binary complex with the receptor kinase. J Biol Chem 274, 15947-15952.

Kjellen, L., and Lindahl, U. (1991). Proteoglycans: structures and interactions. Annu Rev Biochem 60, 443-475.

Kussie, P.H., Hulmes, J.D., Ludwig, D.L., Patel, S., Navarro, E.C., Seddon, A.P., Giorgio, N.A., and Bohlen, P. (1999). Cloning and functional expression of a human heparanase gene. Biochem Biophys Res Commun 261, 183-187.

Lamanna, W.C., Frese, M.A., Balleininger, M., and Dierks, T. (2008). Sulf loss influences $\mathrm{N}-, 2-\mathrm{O}-$, and 6-O-sulfation of multiple heparan sulfate proteoglycans and modulates fibroblast growth factor signaling. J Biol Chem 283, 27724-27735.

Linhardt, R.J., Galliher, P.M., and Cooney, C.L. (1986). Polysaccharide lyases. Appl Biochem Biotechnol 12, 135-176.

Linhardt, R.J., Turnbull, J.E., Wang, H.M., Loganathan, D., and Gallagher, J.T. (1990). Examination of the substrate specificity of heparin and heparan sulfate lyases. Biochemistry 29, 2611-2617. Lohse, D.L., and Linhardt, R.J. (1992). Purification and characterization of heparin lyases from Flavobacterium heparinum. J Biol Chem 267, 24347-24355.

Lunin, V.V., Li, Y., Linhardt, R.J., Miyazono, H., Kyogashima, M., Kaneko, T., Bell, A.W., and Cygler, M. (2004). High-resolution crystal structure of Arthrobacter aurescens chondroitin AC lyase: an enzyme-substrate complex defines the catalytic mechanism. J Mol Biol 337, 367-386.

Luo, Y., Huang, X., and McKeehan, W.L. (2007). High yield, purity and activity of soluble recombinant Bacteroides thetaiotaomicron GST-heparinase I from Escherichia coli. Arch Biochem Biophys 460, 17-24.

Luo, Y., Ye, S., Kan, M., and McKeehan, W.L. (2006). Control of fibroblast growth factor (FGF) 7- and FGF1-induced mitogenesis and downstream signaling by distinct heparin octasaccharide motifs. J Biol Chem 281, 21052-21061.

Maccarana, M., Sakura, Y., Tawada, A., Yoshida, K., and Lindahl, U. (1996). Domain structure of heparan sulfates from bovine organs. J Biol Chem 271, 17804-17810.

Mayans, O., Scott, M., Connerton, I., Gravesen, T., Benen, J., Visser, J., Pickersgill, R., and Jenkins, J. (1997). Two crystal structures of pectin lyase $\mathrm{A}$ from Aspergillus reveal a $\mathrm{pH}$ driven conformational change and striking divergence in the substrate-binding clefts of pectin and pectate lyases. Structure 5, 677-689.

McCarter, J.D., and Withers, S.G. (1994). Mechanisms of enzymatic glycoside hydrolysis. Curr Opin Struct Biol 4, 885-892.

Moffat, C.F., McLean, M.W., Long, W.F., and Williamson, F.B. (1991). Heparinase II from Flavobacterium heparinum. Action on chemically modified heparins. Eur J Biochem 197, 449-459.

Murshudov, G.N., Vagin, A.A., and Dodson, E.J. (1997). Refinement of macromolecular structures by the maximum-likelihood method. Acta Crystallogr D Biol Crystallogr 53, 240-255.

Nader, H.B., Porcionatto, M.A., Tersariol, I.L., Pinhal, M.A., Oliveira, F.W., Moraes, C.T., and Dietrich, C.P. (1990). Purification and substrate specificity of heparitinase I and heparitinase II from Flavobacterium heparinum. Analyses of the heparin and heparan sulfate degradation products by $13 \mathrm{C}$ NMR spectroscopy. J Biol Chem 265, 16807-16813.

Otwinowski, Z., Minor, W., and Charles W. Carter, Jr. (1997). Processing of X-ray diffraction data collected in oscillation mode. In Methods in Enzymology (Academic Press), pp. 307-326.

Perrimon, N., and Bernfield, M. (2000). Specificities of heparan sulphate proteoglycans in developmental processes. Nature 404, 725-728.

Peter, G. (1987). Alginate-modifying enzymes: A proposed unified mechanism of action for the lyases and epimerases. FEBS Letters 212, 199-202.

Rapraeger, A.C., Krufka, A., and Olwin, B.B. (1991). Requirement of heparan sulfate for bFGF-mediated fibroblast growth and myoblast differentiation. Science 252, 1705-1708.

Ren, L., Qin, X., Cao, X., Wang, L., Bai, F., Bai, G., and Shen, Y. (2011). Structural insight into substrate specificity of human intestinal maltase-glucoamylase. Protein Cell 2, 827-836.

Sasisekharan, R., Moses, M.A., Nugent, M.A., Cooney, C.L., and Langer, R. (1994). Heparinase inhibits neovascularization. Proc Natl Acad Sci U S A 91, 1524-1528. 
Sasisekharan, R., and Venkataraman, G. (2000). Heparin and heparan sulfate: biosynthesis, structure and function. Curr Opin Chem Biol 4, 626-631.

Schneider, T.R., and Sheldrick, G.M. (2002). Substructure solution with SHELXD. Acta Crystallogr D Biol Crystallogr 58, 1772-1779.

Shaya, D., Tocilj, A., Li, Y., Myette, J., Venkataraman, G., Sasisekharan, R., and Cygler, M. (2006). Crystal structure of heparinase II from Pedobacter heparinus and its complex with a disaccharide product. J Biol Chem 281, 15525-15535.

Shaya, D., Zhao, W., Garron, M.L., Xiao, Z., Cui, Q., Zhang, Z., Sulea, T., Linhardt, R.J., and Cygler, M. (2010). Catalytic mechanism of heparinase II investigated by site-directed mutagenesis and the crystal structure with its substrate. J Biol Chem 285, 20051-20061.

Sugahara, K., and Kitagawa, H. (2002). Heparin and heparan sulfate biosynthesis. IUBMB Life 54, 163-175.

Takei, Y., Ozawa, Y., Sato, M., Watanabe, A., and Tabata, T. (2004). Three Drosophila EXT genes shape morphogen gradients through synthesis of heparan sulfate proteoglycans. Development
131, 73-82.

Toyoshima, M., and Nakajima, M. (1999). Human heparanase. Purification, characterization, cloning, and expression. J Biol Chem 274, 24153-24160.

Yayon, A., Klagsbrun, M., Esko, J.D., Leder, P., and Ornitz, D.M. (1991). Cell surface, heparin-like molecules are required for binding of basic fibroblast growth factor to its high affinity receptor. Cell 64, 841-848.

Ye, S., Luo, Y., Lu, W., Jones, R.B., Linhardt, R.J., Capila, I., Toida, T., Kan, M., Pelletier, H., and McKeehan, W.L. (2001). Structural basis for interaction of FGF-1, FGF-2, and FGF-7 with different heparan sulfate motifs. Biochemistry 40, 14429-14439.

Yip, V.L., and Withers, S.G. (2004). Nature's many mechanisms for the degradation of oligosaccharides. Org Biomol Chem 2, 2707-2713.

Zhang, F., Zhang, Z., Lin, X., Beenken, A., Eliseenkova, A.V., Mohammadi, M., and Linhardt, R.J. (2009). Compositional analysis of heparin/heparan sulfate interacting with fibroblast growth factor.fibroblast growth factor receptor complexes. Biochemistry 48, 8379-8386. 